\title{
Evaluation of Lumbar Stiffness after Long-Level Fusion for Degenerative Lumbar Scoliosis in Chinese population
}

\section{Xinling Zhang}

Peking University Third Hospital

\section{Lei Yuan}

Peking University Third Hospital

\section{Yan Zeng ( $\sim$ zyanby73@163.com )}

Peking University Third Hospital

\section{Zhongqiang Chen}

Peking University Third Hospital

Weishi Li

Peking University Third Hospital

\section{Chuiguo Sun}

Peking University Third Hospital

\section{Woquan Zhong}

Peking University Third Hospital

\section{Research article}

Keywords: Degenerative lumbar scoliosis, long-level fusion surgery, lumbar stiffness, C-LSDI, ADL

Posted Date: October 28th, 2020

DOl: https://doi.org/10.21203/rs.3.rs-24904/v2

License: (9) This work is licensed under a Creative Commons Attribution 4.0 International License. Read Full License 


\section{Abstract}

Study Design: a retrospective study.

Summary of Background Data: Long-level spinal instrumented fusion for DLS by intention eliminated spinal motion in an attempt to alleviate pain, improve deformity, and reduce disability. However, this surgery considerably impaired performance of activities of daily living (ADL) due to the resulting stiffness. The lumbar stiffness disability index (LSDI) was a validated measure of the effect of lumbar stiffness on functional activity, however, which might not be fully applicable to elderly Chinese population because of their several special lifestyles.

Objective: To evaluate the lumbar stiffness in patients with degenerative lumbar scoliosis (DLS) after long-level fusion by Chinese-LSDI (C-LSDI).

Methods: 129 DLS patients underwent long-level ( $\geqq 4$ levels) fusion surgery with at least one-year followup were included. The C-LSDI was designed by modifying LSDI and Korean-LSDI (K-LSDI) considering elderly Chinese lifestyles, and the patient-reported outcome questionnaire measuring the impact of lumbar stiffness on functional abilities in elderly Chinese with DLS was assessed for internal consistency and retest repeatability.

Results: All patients showed increased lumbar stiffness with significantly improvement in pain and deformity postoperatively, and for items of performing personal hygiene after toileting and getting out of a car, people performed more inconvenient with increasing fixed levels. Compared with LSDI and K-LSDI, the C-LSDI demonstrated high internal consistency (Cronbach's alpha $=0.902)$ and retest reliability $(I C C=0.904)$ in the elderly Chinese population.

Conclusion: This study demonstrated that the C-LSDI questionnaire was a reliable and valid instrument for assessing functional limitations due to lumbar stiffness among elderly Chinese patients with DLS after long-level fusion. Although the effects of stiffness did trend toward greater impacts among patients underwent longer fusions, most patients were satisfied with trade-offs of function and pain relief in exchange for perceived increases in lumbar stiffness.

\section{Introduction}

Degenerative lumbar scoliosis (DLS) is a sort of adult spinal deformity (ASD) disease associated with degeneration of lumbar spine. The prevalence of DLS in Chinese Han population above 40 years old is around $13.3 \%[1]$. Patients with the Cobb angle $\geq 20^{\circ}$ exhibit significant scoliosis and often needed correction surgery with long-level fusion[2,1,3,4]. Lumbar arthrodesis by intention reduces spinal motion in an attempt to decrease pain, deformity, and instability[5]. With increasing numbers of levels fused, however, the resulting loss of lumbar motion had been shown in some patients to impact ability to perform certain activities of daily living(ADL), such as bathing, toileting[6], transferring, and dressing[5]. 
Despite this, there were limited data measuring the functional effects of loss of lumbar mobility after long-level fusion.

Although several self-reported outcomes tools specifically designed for patients who had lumbar spinal surgery were available, they primarily measured pain levels, general health status, and global functioning, and did not address limitations due to spinal stiffness[7-12]. Activities of daily living included bathing, ambulating, toileting, transferring, eating, and dressing. Requirements of lumbar spinal mobility for performance of routine ADL were not well documented, advising patients regarding the likely impacts of lumbar fusion on specific functional activities was difficult.

In an effort to provide objective data regarding expected functional limitations after arthrodesis, Hart et al[13] developed and validated the lumbar stiffness disability index (LSDI) as a patient-reported measure of the effects of stiffness on ability to perform various $A D L$, and then the index was used in several clinical studies to evaluate lumbar mobility after arthrodesis[13-15]. Choi et al[16] designed a modified LSDI considering Korean life style (have a floor-living lifestyle without the use of a bed or a chair, have defecating and toileting in a squatting posture, and have worked in a squatted or crouched posture for agricultural work or household work for a long time). Due to characteristics of elderly Chinese lifestyles, neither the need of self-drive (more than $35 \%$ said they have no need for self-drive), nor the lifestyle of floor living, but the need to bend over housework, maybe neither of these two versions could be used to evaluate lumbar stiffness in Chinese elderly perfectly. This study described the contents of a modified LSDI (Chinese version LSDI, C-LSDI), considering elderly Chinese lifestyles, and an assessment of its internal consistency and test-retest reliability, the key point was the study evaluated its clinical outcome in patients of Chinese population with DLS after long-level fusion.

\section{Materials And Methods}

Our study was approved by ethical review board of our institution. We retrospectively reviewed DLS patients underwent long-level fusion surgery from June 2009 to September 2017 in our hospital. Inclusion criteria were: 1) $\geq 4$ (0years; 2 ) $\geq 4$ fused levels; 3 ) isolated posterior instrumented arthrodesis; 4) minimum 1-year follow up; Exclusion criteria were: 1) other pathology of scoliosis, including idiopathic scoliosis, traumatic scoliosis, ankylosing spondylitis, tuberculosis and tumor; 2) previous lumbar instrumented surgery; 3 ) severe postoperative complications; 4) severe hip and knee joint diseases or severe low back pain caused by other reasons. Totally 129 patients were included in our study. There were 26 males and 103 females. The mean age was 62.8 years (range: $40-79$ years), and the mean BMI was $25.2 \mathrm{~kg} / \mathrm{m} 2$ (range: $17-35 \mathrm{~kg} / \mathrm{m} 2$ ).

The general information was collected by electronic medical record system in our hospital, including age, sex, and body mass index (BMI). The surgery-related data including upper instrumented vertebrae (UIV), lower instrumented vertebrae (LIV), fusion to S1, and fixed levels. Two orthopedic medical doctors measured imaging parameters separately by Centricity Enterprise Web V3.0, all patients underwent the standard full-back spine X-Ray[17]. The following radiographic parameters were measured preoperatively, 
and the final follow-up: Cobb, apical vertebral translation (AVT), coronal vertical axis (CVA), sagittal vertical axis (SVA), thoracic kyphosis (TK), thoracolumbar kyphosis (TLK), pelvic tilt (PT), pelvic incidence (PI), sacral slope (SS), lumbar lordosis (LL), and PI-LL.

We designed a modified Chinese version of LSDI (C-LSDI) considering the elderly Chinese lifestyle. Through the follow-up of Chinese population with DLS, high follow-up loss rate performed in question 3 (Drive a motor vehicle) and question 10 (Engage in sexual intercourse) in LSDI and question 5 (Use squatting toilet) in K-LSDI. The intraclass correlation coefficient (ICC) of question 7 (Sit cross-legged on the floor and get up) in K-LSDI was poor. Therefore, in the C-LSDI, these questions were deleted, and question 7 (Bend forward to clean the floor) (DLS was more common in elder female and the need to bend over housework) and question 11 (Take a small object sideways on your own) (Reflect the rotation of spine) were added (Table 1). Patients were asked to complete the VAS, ODI, JOA29, SRS22, SF36 and C-LSDI preoperatively and at 1 years after surgery. Patients who did not complete the questionnaires, or who were unable to attend the follow-up were contacted by research staff and asked to complete the questionnaires by phone.

\section{Statistical Analysis}

Statistical analysis was conducted with SPSS 22.0 (IBM Corp., USA). Independent t-test was performed to compare consecutive variables between independent groups, and paired t-test was performed to compare consecutive pre-and post-operative variables. Linear regression models were used to identify the relation between fixed levels and C-LSDI scores.

\section{Internal consistency}

We computed the Cronbach alpha statistic, which measured the degree of internal consistency of response items represented by a scale or score. Cronbach alpha values ranged from 0 to 1 , with higher values indicating greater correlation among the questionnaire items. High internal consistency indicated that patients with higher scores on one question also tend to score higher on other questions, that was, the questions assessed related functional domains. For clinical studies, a Cronbach alpha value of 0.9 or greater was ideal, whereas 0.7 or greater was considered satisfactory[18,19].

\section{Retest reliability}

To assess retest repeatability, we computed an intraclass correlation coefficient (ICC), a measurement of the reproducibility of repeated measures of the same patient. Values of the ICC ranged from 0 to 1; values of 0.7 or greater indicated excellent agreement[20].

\section{Results}

\section{Internal consistency and retest reliability of C-LSDI}


Table 1. The internal consistency of LSDI, K-LSDI and C-LSDI

\begin{tabular}{|c|c|c|}
\hline LSDI & K-LSDI & C-LSDI \\
\hline $\begin{array}{l}\text { 1. Bend to your feet to put on } \\
\text { your underwear and pants } \\
\text { while dressing independently }\end{array}$ & $\begin{array}{l}\text { 1. Bend to your feet to put on } \\
\text { your underwear, pants and } \\
\text { socks on your own }\end{array}$ & $\begin{array}{l}\text { 1.Bend to your feet to put on } \\
\text { your underwear and pants on } \\
\text { your own }\end{array}$ \\
\hline $\begin{array}{l}\text { 2. Bend through your waist to } \\
\text { put on your socks and shoes } \\
\text { 3. Drive a motor vehicle }\end{array}$ & $\begin{array}{l}\text { 2. Bend forward to pick up a } \\
\text { small object on the floor on } \\
\text { your own }\end{array}$ & $\begin{array}{l}\text { 2.Bend to your feet to put on } \\
\text { your socks and shoes on your } \\
\text { own }\end{array}$ \\
\hline $\begin{array}{l}\text { 4. Perform personal hygiene } \\
\text { functions following toileting } \\
\text { 5. Bend forward to pick up a }\end{array}$ & $\begin{array}{l}\text { 3. Bend forward to wash your } \\
\text { face and hair without } \\
\text { getting wet }\end{array}$ & $\begin{array}{l}\text { 3. Bend forward to pick up a } \\
\text { small object on the floor on } \\
\text { your own }\end{array}$ \\
\hline small object off the floor & 4. Bathe the lower half of your & 4. Bend forward to wash your \\
\hline 6. Get in and out of bed & & face and hair without getting \\
\hline $\begin{array}{l}\text { 7. Get in and out of a chair } \\
8 \text {. Bathe the lower half of your } \\
\text { body }\end{array}$ & $\begin{array}{l}\text { 5. Use conventional floor toilet } \\
\text { sent on your own } \\
\text { 6. Wipe anus with hand after }\end{array}$ & $\begin{array}{l}\text { wet } \\
5 \text {. Bathe the lower half of your } \\
\text { body on your own }\end{array}$ \\
\hline $\begin{array}{l}\text { 9.Get in and out of an } \\
\text { automobile } \\
\text { 10. Engage in sexual intercourse }\end{array}$ & $\begin{array}{l}\text { defecation } \\
\text { 7. Sit crossed-legged on the } \\
\text { floor and get up on your } \\
\text { own }\end{array}$ & $\begin{array}{l}\text { 6.Perform personal hygiene } \\
\text { functions following toileting } \\
\text { 7.Bend forward to clean the } \\
\text { floor on your own }\end{array}$ \\
\hline $\begin{array}{l}\text { Response options and score } \\
\text { for each item }\end{array}$ & $\begin{array}{l}\text { 8. Lie down on the bed and get } \\
\text { up on your own }\end{array}$ & $\begin{array}{l}\text { 8.Sit down on the chair and } \\
\text { get up on your own }\end{array}$ \\
\hline $\begin{array}{l}0 \text { No effect at all } \\
1 \text { Minor effect }\end{array}$ & $\begin{array}{l}\text { 9. Sit down on the chair and } \\
\text { get up on your own }\end{array}$ & $\begin{array}{l}\text { 9. Lie down on the bed and } \\
\text { get up on your own }\end{array}$ \\
\hline $\begin{array}{l}2 \text { Significant effect } \\
3 \text { Require assistance }\end{array}$ & $\begin{array}{l}\text { 10. Get in and out of an } \\
\text { automobile on your own }\end{array}$ & $\begin{array}{l}\text { 10. Get in and out of a car on } \\
\text { your own }\end{array}$ \\
\hline \multirow[t]{5}{*}{4 Cannot do at all } & $\begin{array}{l}\text { 11. Engage in sexual intercourse } \\
\text { Response options and score for } \\
\text { each item } \\
0 \text { No effect at all }\end{array}$ & $\begin{array}{l}\text { 11.Take a small object } \\
\text { sideways on your own } \\
\text { Response options and score } \\
\text { for each item }\end{array}$ \\
\hline & $\begin{array}{l}1 \text { Help oneself but have minor } \\
\text { effect } \\
2 \text { Help oneself but have effect }\end{array}$ & $\begin{array}{l}0 \text { No effect at all } \\
1 \text { Help oneself but have minor } \\
\text { effect }\end{array}$ \\
\hline & $\begin{array}{l}3 \text { Help oneself but have } \\
\text { significant effect } \\
4 \text { Cannot help oneself require } \\
\text { assistance }\end{array}$ & $\begin{array}{l}2 \text { Help oneself but have effect } \\
3 \text { Help oneself but have } \\
\text { significant effect } \\
4 \text { Cannot help oneself require }\end{array}$ \\
\hline & 5 Cannot do at all & assistance \\
\hline & & 5 Cannot do at all \\
\hline Cronbach alpha $=0.758$ & Cronbach alpha $=0.852$ & Cronbach alpha $=0.902$ \\
\hline
\end{tabular}

The follow-up loss rate of question 3 and question 10 in LSDI was $35.7 \%$ and $79.8 \%$ in Chinese population, respectively. The follow-up loss rate of question 5 in K-LSDI was $33.3 \%$ in Chinese population. Therefor, the C-LSDI deleted the question 3 and 10 in LSDI, and the question 5 and 7 in K-LSDI, adding question 7 and 11.

LSDI: Lumbar Stiffness Disability Index; K-LSDI: Korean-LSDI; C-LSDI: Chinese-LSDI.

The ICC of driving and sexual intercourse in LSDI and sitting cross-legged in K-LSDI were poor, with other items in LSDI and K-LSDI were great. Each item in C-LSDI demonstrated great ICC. The total score of CLSDI (ICC=0.904) showed better reliability than LSDI (ICC=0.862) and K-LSDI (ICC=0.887) (Table 3). 
Table 3. The ICC of LSDI, K-LSD and C-LSDI

\begin{tabular}{|c|c|}
\hline Item & $\mathrm{ICC}$ \\
\hline 1.Bend to your feet to put on your underwear and pants on your own & $0.763^{*}$ \\
\hline 2.Bend to your feet to put on your socks and shoes on your own & $0.822 *$ \\
\hline 3. Drive a motor vehicle & 0.651 \\
\hline 4.Perform personal hygiene functions following toileting & $0.788^{*}$ \\
\hline 5.Bend forward to pick up a small object on the floor on your own & $0.752^{*}$ \\
\hline 6. Lie down on the bed and get up on your own & $0.775^{*}$ \\
\hline 7.Sit down on the chair and get up on your own & $0.868^{*}$ \\
\hline 8. Bathe the lower half of your body on your own & $0.705^{*}$ \\
\hline 9.Get in and out of a car on your own & $0.784^{*}$ \\
\hline 10. Engage in sexual intercourse & 0.506 \\
\hline $\begin{array}{l}\text { 11.Bend forward to wash your face and hair without getting wet } \\
\text { (K-LSDI) (C-LSDI) }\end{array}$ & $0.798^{*}$ \\
\hline 12.Use conventional floor toilet sent on your own(K-LSDI) & $0.802^{*}$ \\
\hline 13.Sit crossed-legged on the floor and get up on your own(K-LSDI) & 0.429 \\
\hline 14.Bend forward to clean the floor on your own(C-LSDI) & $0.870^{*}$ \\
\hline 15.Take a small object sideways on your own(C-LSDI) & $0.709^{*}$ \\
\hline LSDI scores & $0.862^{*}$ \\
\hline K-LSDI scores & $0.887^{*}$ \\
\hline C-LSDI scores & $0.904^{*}$ \\
\hline
\end{tabular}

The LSDI scale contained question 1-10.

${ }^{*} \mathrm{ICC}>0.7$.

The ICC value was between 0 and 1 . Generally, the reliability was considered poor if ICC $<0.4$, and good if ICC >0.7.

\section{Deformity correction and clinical results}

The Cobb angle, AVT, and PT decreased significantly, and SS and TK increased significantly, postoperatively. There was no significant difference in CVA, SVA, TLK, PI, LL and PI-LL between preoperation and follow-up (Table 2). 


\begin{tabular}{llll}
\hline & preoperative & postoperative & \multicolumn{1}{c}{ P value } \\
\hline Cobb $\left({ }^{\circ}\right)$ & $30.2 \pm 11.6$ & $11.6 \pm 6.9$ & $<0.0001^{*}$ \\
AVT $(\mathrm{mm})$ & $27.7 \pm 13.8$ & $15.8 \pm 9.4$ & $<0.0001^{*}$ \\
CVA $(\mathrm{mm})$ & $18.1 \pm 17.6$ & $18.6 \pm 13.4$ & 0.755 \\
SVA $(\mathrm{mm})$ & $51.2 \pm 51.2$ & $51.8 \pm 39.4$ & 0.880 \\
TK $\left({ }^{\circ}\right)$ & $19.0 \pm 14.8$ & $24.8 \pm 13.4$ & $<0.0001^{*}$ \\
TLK $\left({ }^{\circ}\right)$ & $14.3 \pm 19.0$ & $11.8 \pm 12.1$ & 0.080 \\
PT $\left({ }^{\circ}\right)$ & $23.9 \pm 9.9$ & $21.2 \pm 12.0$ & $\mathbf{0 . 0 0 2 ^ { * }}$ \\
PI $\left({ }^{\circ}\right)$ & $49.5 \pm 11.3$ & $49.8 \pm 10.9$ & 0.081 \\
SS $\left({ }^{\circ}\right)$ & $25.5 \pm 10.9$ & $27.0 \pm 10.3$ & $\mathbf{0 . 0 2 2 ^ { * }}$ \\
LL $\left({ }^{\circ}\right)$ & $32.5 \pm 58.9$ & $33.4 \pm 14.5$ & 0.907 \\
PI-LL $\left({ }^{\circ}\right)$ & $16.7 \pm 57.7$ & $16.6 \pm 14.5$ & 0.960 \\
\hline
\end{tabular}

${ }^{*} \mathrm{p}<0.01$.

AVT: apical vertebral translation, CVA: coronal vertical axis, SAV: sagittal vertical axis, TK: thoracic kyphosis, TLK: thoracolumbar kyphosis, PT: pelvic tilt, PI: pelvic incidence, SS: sacral slope, LL: lumbar lordosis.

There were significant improvement in post-operation clinical symptoms reflected in the following scales: VAS for back and low extremity pain, ODI, JOA, SRS-22 including pain, self-image, psychological status and activity ability, SF-36 involving physical functioning (PF), role-physical (RP), bodily pain (BP), general health (GH), vitality (VT), social functioning (SF), role-emotional (RE), mental health (MH), physical component scores (PCS) and mental component scores (MCS). The change in C-LSDI scores increased postoperatively, indicating increasing limitations due to stiffness after longer arthrodesis. Interestingly, CLSDI scores did not correlate with patient's satisfaction, overall, they were satisfied with the surgery with mean satisfactory score was 3.42 (total score 5 ).

Simple linear regression model demonstrated that fixed levels and several items in C-LSDI were positively correlated, included washing lower body, performing perineal hygiene after toileting, cleaning floor, getting out of chair, getting out of bed and getting out of car. The scores of question 6 (perform peroneal hygiene after toileting) and question 10 (get out of car) in C-LSDI showed that with increasing fixed levels, patients behaved significant stiffness (Table 6, Figure 1). 


\begin{tabular}{|c|c|c|c|c|}
\hline & & Preoperative & Postoperative & $\begin{array}{c}P \\
\text { value }\end{array}$ \\
\hline \multicolumn{2}{|c|}{ VAS (low back) } & $6.4 \pm 1.8$ & $3.0 \pm 2.1$ & $<0.0001 *$ \\
\hline \multicolumn{2}{|l|}{ VAS (leg) } & $5.6 \pm 2.2$ & $2.3 \pm 2.0$ & $<0.0001^{*}$ \\
\hline \multicolumn{2}{|l|}{ ODI } & $61.3 \pm 37.6$ & $26.5 \pm 18.7$ & $<0.0001 *$ \\
\hline \multicolumn{2}{|l|}{ JOA-29 } & $11.4 \pm 3.8$ & $20.6 \pm 5.6$ & $<0.0001 *$ \\
\hline \multirow[t]{5}{*}{ SRS-22 } & pain & $2.4 \pm 1.0$ & $4.1 \pm 0.9$ & $<0.0001^{*}$ \\
\hline & Self-image & $2.2 \pm 0.6$ & $4.0 \pm 0.8$ & $<0.0001 *$ \\
\hline & Mental health & $2.5 \pm 0.7$ & $4.1 \pm 0.8$ & $<0.0001^{*}$ \\
\hline & Activity & $2.3 \pm 0.7$ & $3.6 \pm 0.8$ & $<0.0001 *$ \\
\hline & Satisfaction of management & & $3.4 \pm 0.7$ & \\
\hline \multirow[t]{8}{*}{ SF-36 } & Physical Functioning, PF & $33.3 \pm 17.3$ & $74.3 \pm 19.2$ & $<0.0001 *$ \\
\hline & Role-Physical, RP & $2.1 \pm 11.3$ & $50.6 \pm 47.9$ & $<0.0001^{*}$ \\
\hline & Bodily Pain, BP & $32.4 \pm 20.5$ & $71.7 \pm 18.3$ & $<0.0001 *$ \\
\hline & General Health, GH & $31.4 \pm 20.2$ & $70.9 \pm 21.9$ & $<0.0001 *$ \\
\hline & Vitality, VT & $46.0 \pm 19.2$ & $76.3 \pm 17.4$ & $<0.0001^{*}$ \\
\hline & Social Functioning, SF & $35.5 \pm 23.5$ & $71.1 \pm 22.7$ & $<0.0001^{*}$ \\
\hline & Role-Emotional, RE & $9.5 \pm 26.0$ & $64.6 \pm 47.8$ & $<0.0001^{*}$ \\
\hline & Mental Health, $\mathrm{MH}$ & $44.7 \pm 22.9$ & $73.7 \pm 17.2$ & $<0.0001^{*}$ \\
\hline \multicolumn{2}{|l|}{ PCS } & $17.2 \pm 7.7$ & $43.9 \pm 12.8$ & $<0.0001^{*}$ \\
\hline \multicolumn{2}{|l|}{$\operatorname{MCS}$} & $35.6 \pm 11.0$ & $50.8 \pm 10.9$ & $<0.0001^{*}$ \\
\hline \multirow[t]{11}{*}{ C-LSDI } & $\begin{array}{l}\text { 1.Bend to your feet to put on your } \\
\text { underwear and pants on your own }\end{array}$ & $1.8 \pm 0.9$ & $3.3 \pm 1.1$ & $<0.0001^{*}$ \\
\hline & $\begin{array}{l}\text { 2.Bend to your feet to put on your socks and } \\
\text { shoes on your own }\end{array}$ & $2.4 \pm 0.9$ & $3.5 \pm 1.3$ & $<0.0001^{*}$ \\
\hline & $\begin{array}{l}\text { 3.Bend forward to pick up a small object on } \\
\text { the floor on your own }\end{array}$ & $2.2 \pm 0.8$ & $3.8 \pm 1.2$ & $<0.0001 *$ \\
\hline & $\begin{array}{l}\text { 4.Bend forward to wash your face and hair } \\
\text { without getting wet }\end{array}$ & $1.3 \pm 0.5$ & $3.2 \pm 1.1$ & $<0.0001 *$ \\
\hline & $\begin{array}{l}\text { 5.Bathe the lower half of your body on your } \\
\text { own }\end{array}$ & $2.7 \pm 0.8$ & $3.6 \pm 1.3$ & $<0.0001 *$ \\
\hline & $\begin{array}{l}\text { 6.Perform personal hygiene functions } \\
\text { following toileting }\end{array}$ & $1.6 \pm 0.6$ & $3.6 \pm 1.4$ & $<0.0001^{*}$ \\
\hline & $\begin{array}{l}\text { 7.Bend forward to clean the floor on your } \\
\text { own }\end{array}$ & $2.9 \pm 0.6$ & $3.4 \pm 1.2$ & $<0.0001 *$ \\
\hline & $\begin{array}{l}\text { 8.Sit down on the chair and get up on your } \\
\text { own }\end{array}$ & $2.1 \pm 0.9$ & $2.5 \pm 1.1$ & $0.0020^{*}$ \\
\hline & $\begin{array}{l}\text { 9.Lie down on the bed and get up on your } \\
\text { own }\end{array}$ & $2.1 \pm 0.7$ & $2.6 \pm 1.2$ & $<0.0001^{*}$ \\
\hline & 10.Get in and out of a car on your own & $1.8 \pm 0.7$ & $3.7 \pm 1.4$ & $<0.0001^{*}$ \\
\hline & 11.Take a small object sideways on your own & $1.5 \pm 1.1$ & $3.6 \pm 1.2$ & $<0.0001^{*}$ \\
\hline
\end{tabular}

$* p<0.01$.

PCS: physical component scores; MCS: mental component scores. 


\begin{tabular}{lll}
\hline & & $P$ value \\
\hline UIV & $56(43.4 \%)$ & \\
G1 ( $\geq$ T11) & $61(47.3 \%)$ & 0.178 \\
G2 (T12-L1) & $12(9.3 \%)$ & \\
G3 (<L1) & & \\
IIV & $50(38.8 \%)$ & 0.230 \\
Fusion to S1 & $79(61.2 \%)$ & \\
Fusion to L5 or above & $33(25.6 \%)$ & \\
IFL & $46(35.7 \%)$ & \\
0 & $39(30.2 \%)$ & 0.423 \\
1 & $9(7.0 \%)$ & \\
2 & $2(1.5 \%)$ & \\
3 & & \\
4 & $12(9.3 \%)$ & \\
Osteotomy type & $58(45.0 \%)$ & \\
O(No osteotomy) & $44(34.1 \%)$ & \\
1 & $13(10.0 \%)$ & \\
2 & $1(7.7 \%)$ & \\
3 & & \\
4 & & \\
\hline
\end{tabular}

UIV: upper instrumented vertebrae; LIV: lower instrumented vertebrae; IFL: intervertebral fusion level.

Table 6. Linear correlation between fixed levels and the change scores of C-LSDI

\begin{tabular}{|c|c|c|}
\hline C-LSDI & $P$ value & Forecast formula \\
\hline Total score & $0.033^{*}$ & $Y=1.289 \times X+7.027$ \\
\hline $\begin{array}{l}\text { 1.Bend to your feet to put on your } \\
\text { underwear and pants on your own }\end{array}$ & 0.528 & \\
\hline $\begin{array}{l}\text { 2.Bend to your feet to put on your } \\
\text { socks and shoes on your own }\end{array}$ & 0.225 & \\
\hline $\begin{array}{l}\text { 3.Bend forward to pick up a small } \\
\text { object on the floor on your own }\end{array}$ & 0.489 & \\
\hline $\begin{array}{l}\text { 4. Bend forward to wash your face } \\
\text { and hair without getting wet }\end{array}$ & 0.422 & \\
\hline $\begin{array}{l}\text { 5. Bathe the lower half of your body } \\
\text { on your own }\end{array}$ & $0.006^{* *}$ & $Y=0.200 \times X-0.286$ \\
\hline $\begin{array}{l}\text { 6. Perform personal hygiene } \\
\text { functions following toileting }\end{array}$ & $0.003^{* *}$ & $Y=0.208 \times X+0.771$ \\
\hline $\begin{array}{l}\text { 7.Bend forward to clean the floor on } \\
\text { your own }\end{array}$ & $0.021^{*}$ & $Y=0.149 \times X-0.354$ \\
\hline $\begin{array}{l}\text { 8. Sit down on the chair and get up } \\
\text { on your own }\end{array}$ & $0.0497^{*}$ & $Y=0.136 \times X-0.415$ \\
\hline $\begin{array}{l}\text { 9.Lie down on the bed and get up on } \\
\text { your own }\end{array}$ & $0.022^{*}$ & $Y=0.144 \times X-0.366$ \\
\hline $\begin{array}{l}\text { 10.Get in and out of a car on your } \\
\text { own }\end{array}$ & $0.021^{*}$ & $Y=0.169 \times X+0.944$ \\
\hline $\begin{array}{l}\text { 11.Take a small object sideways on } \\
\text { your own }\end{array}$ & 0.164 & \\
\hline
\end{tabular}

${ }^{*} p<0.05,{ }^{* *} p<0.01$.

$\mathrm{Y}=$ change scores of C-LSDI postoperatively ; $\mathrm{X}=$ fixed levels 
This study demonstrated that patients with DLS enjoyed significant improvement in pain, physical function and deformity after long-level fusion, which was similar to previous studies. Patients with high levels of disability due to sagittal imbalance or spinal stenosis had been demonstrated to experience substantial benefit from total lumbar arthrodesis surgery as compared with nonoperative treatment with respect to reductions in leg and back pain and improvements in physical function[21,14,22-25]. However, despite the benefits of correction surgery, extensive spinal fusion also resulted in limited flexibility and to some extent impaired ADL, especially for elderly patients[13].

Stiffness, liked pain, was to some extent a subjective complaint. Although it was possible to measure actual range of motion using radiography[10] or other motion tracking techniques, such approaches did not necessarily informed us of patients' perceptions of their flexibility, and more specifically of any effects of stiffness on their everyday functional abilities. A self-reported questionnaire such as the LSDI remained the best clinical tool for such an assessment[26].

Previous studies revealed that elder and female patients might be more susceptible to the development of DLS in Chinese Han population[1]. Elderly female in China might face more need for household chores, rather than self-driving, and compared with Korean population, we were also different from their floorliving lifestyle without the use of a bed or a chair. Given the above states, we designed C-LSDI by modifying LSDI and K-LSDI. In this study group of 129 Chinese adult lumbar arthrodesis patients, C-LSDI demonstrated excellent internal consistency and retest reliability compared with LSDI and K-LSDI, several shortcomings must be considered when interpreting the data, in order to solve the matter of linguistic discrepancies, we translated LSDI and K-LSDI into Chinese, which might introduce bias as data collecting.

Our data showed that increased length of lumbar fusions seemed to increase the stiffness that impaired their ability to wash lower body, perform perineal hygiene after toileting, clean floor, get out of chair, get out of bed and get out of car, and of these disabilities, some patients had significant difficulty in doing perineal self-care functions because of limited range of motion after extended fusion. Bafus et al[6] observed increased difficulties with perineal care compared with their preoperative functional ability were reported by five of the14 patients (36\%) having thoracolumbar fusions to L5 or to the sacrum. The result might indicate activities, the higher requirement for flexibility, the more vulnerable.

Another issue was the potential differences in impact of stiffness on ADL after instrumented total lumbar fusions to S1; specifically, between patients with UIV within the upper thoracic (UT) versus the thoracolumbar (TL) region. Sciubba et al[14] defined cohort based on the UIV as UT (T1-T6) or TL (T9L1), and found UT had greater impairment in performing hygiene after toileting. Choi et al[16] categorized patients into 2 group based on UIV, group 1 was above T10 of UIV, group 2 was L1 or L2, and observed group 1 showed higher score compared to group 2 except for question 11(sexual intercourse) in K-LSDI. Interestingly, while patients fused to the upper thoracic spine reported higher mean C-LSDI scores than patients with thoracolumbar stopping points, this did not reach statistical significance in our study. There might be structural or radiographic aspects of patients' preoperative deformity which lead surgeon to choose upper thoracic versus thoracolumbar endpoints, but we were unable to assess this question in the 
current analysis. The functional significance of isolated L5-S1 motion still was not completely defined. Given the relatively small sample size, our study did not allow meaningful statistical comparisons, which was similar with previous study, Bafus et al[6] found that the incidence of perineal care difficulties was similar between patients having fusion to $L 5$ and patients having fusion to the sacrum, maintenance of L5-S1 segmental motion did not seem to reduce occurrence of perineal care problems.

In a cohort of patients with an average of 10 motion segments fused, Wilk et al[27] used a tool of computerized movement analysis and found that compared to normal subjects and unfused patients, patients who had lumbar fusions extending to L3 or L4 had $36 \%$ less total lumbar motion $(P<0.05)$. However, Winter et al[28] observed that as the distal level of fusion moved caudally from T12 to L4, there was no significant change in functional spinal motion until the construct extended to L4. The above discrepancy indicated the difference between instrument-assisted methods and patient-reported outcome tools.

Data collection from 129 patients with DLS after long-level fusion, Patients were satisfied with the arthrodesis procedure, which was similar to previous study. Hart et al[29] showed overall among the entire cohort, $46 \%$ (27/59) of patients indicated that they experienced significant limitations in daily activity due to back stiffness, however, a large majority of patients indicated that they would undergo the same procedure again $(97 \%, 55 / 57)$ and thought that any increase in stiffness from the surgery was an acceptable trade-off for overall functional improvement $(91 \%, 52 / 57)$.

Although these results demonstrated sufficiently strong validity and reliability of the C-LSDI to support its use in the clinical outcome research, prospective application of the tool was needed to establish whether reported functional limitations were truly a result of the arthrodesis procedure. The data presented here could not be interpreted as showing that increased limitations were caused by the fusion because this was a retrospective cohort. The association between pain and spinal stiffness was

complex[30,15], it was possible that patients' answers regarding the functional domains of C-LSDI might be affected by issues other than lumbar stiffness, such as low back pain or hip and knee pathology[15]. Rather, the intent of this study was to establish the validity and psychometric properties of the C-LSDI. Further work to assess the minimum clinically important differences in C-LSDI scores as well as the relative importance of the C-LSDI to patients in comparison with the current pain and deformity-related outcome will also be helpful to address patient questions regarding these potential impacts from surgery[31].

One limitation of our study was that we had not made measurements of our patients' actual lumbar range of motion to establish a true "gold standard." However, prior work had established that the LSDI did indeed correlate to radiographic measurements of lumbar mobility[13]. Despite this, the study had certain advantages. This study expanded on prior work demonstrating the validity and utility of the C-LSDI in assessing stiffness impacts among Chinese patients, and this study also focuses on a single diagnostic and surgical treatment category, i.e., patients with DLS underwent long-level fusion. These data might enhance understanding of functional outcome measurements related to spinal stiffness in patients with 
DLS before arthrodesis, and might allow for more informed preoperative counseling to patients indicated for such surgical interventions.

\section{Conclusion}

The C-LSDI scoring system was useful as a measure of ADL limitations in Chinese lifestyle. In the current study we examined the lumbar stiffness in Chinese population with DLS who underwent long-level fusion with C-LSDI, which had excellent validity and reliability, and found difficulty in performing certain ADL attributed to stiffness increases with increasing levels of lumbar arthrodesis, Fortunately, a large majority reported that such limitations were a satisfactory trade-off for their postoperative pain relief and functional improvements. Although these results might not change spine surgeons' surgical plan or technique for a given patient, we hope they would be useful in the informed consent process to answer patient concerns regarding the expected effect of a long-level fusion and resulting loss of lumbar range of motion on daily activities.

\section{Abbreviations}

DLS: degenerative lumbar scoliosis;

ASD: adult spinal deformity;

ADL: activities of daily living;

LSDI: lumbar stiffness disability index;

BMI :body mass index;

UIV: upper instrumented vertebrae;

LIV: lower instrumented vertebrae;

AVT: apical vertebral translation;

CVA: coronal vertical axis;

SAV: sagittal vertical axis;

TK: thoracic kyphosis;

TLK: thoracolumbar kyphosis;

PT: pelvic tilt;

PI: pelvic incidence; 
SS: sacral slope, LL: lumbar lordosis;

ICC: intraclass correlation coefficient;

PF: physical functioning;

RP: role-physical;

BP: bodily pain;

$\mathrm{GH}$ : general health;

VT: vitality;

SF: social functioning;

RE: role-emotional;

MH: mental health;

PCS: physical component scores;

MCS: mental component scores;

UT: upper thoracic;

TL: thoracolumbar

\section{Declarations}

\section{Availability of data and materials}

The datasets used and/or analyzed for the current study are available from the corresponding author on reasonable request.

\section{Acknowledgements}

None

\section{Funding}

This work did not receive any financial support.

\section{Author information}

Xinling Zhang contributed mainly to the work and should be considered first author. 


\section{Affiliations}

Orthopaedic Department, Peking University Third Hospital, No. 49 North Garden Road, Haidian District, Beijing 100191, China.

Xinling Zhang, Lei Yuan, Yan Zeng, Zhongqiang Chen, Weishi Li, Chuiguo Sun, Woquan Zhong.

\section{Contributions}

XLZ: wrote and prepared the manuscript. LY, ZQC, WSL, CGS and WQZ: data collection and follow-up assessment. YZ: designed study. All authors have read reviewed, and approved the final manuscript.

\section{Correspondence author}

Correspondence to Yan Zeng.

\section{Ethics declarations}

\section{Ethics approval and consent to participate}

This study was approved by the Ethics Committee of Medical Ethics of Peking University Third Hospital, the project number was IRB00006761-M2018076.

\section{Consent for publication}

Not applicable.

\section{Competing interests}

The authors declare that they have no competing interests.

\section{References}

[1] Xu L, Sun X, Huang S, et al. Degenerative lumbar scoliosis in Chinese Han population: prevalence and relationship to age, gender, bone mineral density, and body mass index[J]. Eur Spine J, 2013, 22(6): 13261331. https://doi.org/10.1007/s00586-013-2678-8.

[2] Hong J Y, Suh S W, Modi H N, et al. The prevalence and radiological findings in 1347 elderly patients with scoliosis[J]. J Bone Joint Surg Br, 2010, 92(7): 980-983. https://doi.org/10.1302/0301620X.92B7.23331.

[3] Palmisani M, Dema E, Cervellati S. Surgical treatment of adult degenerative scoliosis[J]. Eur Spine J, 2013, 22 Suppl 6: S829-833. https://doi.org/10.1007/s00586-013-3012-1.

[4] Togawa D, Hasegawa T, Yamato Y, et al. Postoperative Disability After Long Corrective Fusion to the Pelvis in Elderly Patients With Spinal Deformity[J]. Spine (Phila Pa 1976), 2018, 43(14): E804-E812. 
https://doi.org/10.1097/BRS.0000000000002540.

[5] Kane R L, Rockwood T, Philip I, et al. Differences in valuation of functional status components among consumers and professionals in Europe and the United States[J]. Journal of Clinical Epidemiology, 1998, 51(8): 657-666. https://doi.org/10.1016/S0895-4356(98)00038-9.

[6] Bafus T, Shea M, Hart R. Impairment of perineal care functions after long fusions of the lumbar spine[J]. Clin Orthop Relat Res, 2005, \&NA;(433): 111-114.

https://doi.org/10.1097/01.blo.0000150563.31880.0a.

[7] Berven S, Deviren V, Demir-Deviren S, et al. Studies in the modified Scoliosis Research Society Outcomes Instrument in adults: validation, reliability, and discriminatory capacity[J]. Spine (Phila Pa 1976), 2003, 28(18): 2164-2169; discussion 2169. https://doi.org/10.1097/01.brs.0000084666.53553.d6.

[8] Bridwell K H, Cats-Baril W, Harrast J, et al. The validity of the SRS-22 instrument in an adult spinal deformity population compared with the Oswestry and SF-12 - A study of response distribution, concurrent validity, internal consistency, and reliability[J]. Spine, 2005, 30(4): 455-461.

https://doi.org/10.1097/01.brs.0000153393.82368.6b.

[9] Asher M, Min Lai S, Burton D, et al. The reliability and concurrent validity of the scoliosis research society-22 patient questionnaire for idiopathic scoliosis[J]. Spine (Phila Pa 1976), 2003, 28(1): 63-69. https://doi.org/10.1097/00007632-200301010-00015.

[10] Roland M, Fairbank J. The Roland-Morris Disability Questionnaire and the Oswestry Disability Questionnaire[J]. Spine (Phila Pa 1976), 2000, 25(24): 3115-3124. https://doi.org/10.1097/00007632200012150-00006.

[11] Bokshan S L, Godzik J, Dalton J, et al. Reliability of the revised Scoliosis Research Society-22 and Oswestry Disability Index (ODI) questionnaires in adult spinal deformity when administered by telephone[J]. Spine J, 2016, 16(9): 1042-1046. https://doi.org/10.1016/j.spinee.2016.03.022.

[12] Mmopelwa T, Ayhan S, Yuksel S, et al. Analysis of factors affecting baseline SF-36 Mental Component Summary in Adult Spinal Deformity and its impact on surgical outcomes[J]. Acta Orthop Traumatol Turc, 2018, 52(3): 179-184. https://doi.org/10.1016/j.aott.2018.02.001.

[13] Hart R A, Gundle K R, Pro S L, et al. Lumbar Stiffness Disability Index: pilot testing of consistency, reliability, and validity[J]. Spine J, 2013, 13(2): 157-161. https://doi.org/10.1016/j.spinee.2012.12.001.

[14] Sciubba D M, Scheer J K, Smith J S, et al. Which daily functions are most affected by stiffness following total lumbar fusion: comparison of upper thoracic and thoracolumbar proximal endpoints[J]. Spine (Phila Pa 1976), 2015, 40(17): 1338-1344. https://doi.org/10.1097/brs.0000000000000968.

[15] Daniels A H, Smith J S, Hiratzka J, et al. Functional Limitations Due to Lumbar Stiffness in Adults With and Without Spinal Deformity[J]. Spine (Phila Pa 1976), 2015, 40(20): 1599-1604. 
https://doi.org/10.1097/BRS.0000000000001090.

[16] Choi J H, Jang J S, Yoo K S, et al. Functional Limitations Due to Stiffness After Long-Level Spinal Instrumented Fusion Surgery to Correct Lumbar Degenerative Flat Back[J]. Spine (Phila Pa 1976), 2018, 43(15): 1044-1051. https://doi.org/10.1097/BRS.0000000000002514.

[17] Bakhsheshian J, Scheer J K, Gum J L, et al. Comparison of Structural Disease Burden to Healthrelated Quality of Life Scores in 264 Adult Spinal Deformity Patients With 2-Year Follow-up Novel Insights into Drivers of Disability[J]. Clinical Spine Surgery, 2017, 30(2): E124-E131.

https://doi.org/10.1097/BSD.0000000000000470.

[18] Bland J M, Altman D G. Cronbach's alpha[J]. British Medical Journal, 1997, 314(7080): 572-572. https://doi.org/10.1016/j.jmva.2005.05.012.

[19] Vale L, Silcock J, Rawles J. An economic evaluation of thrombolysis in a remote rural community[J]. British Medical Journal, 1997, 314(7080): 570-572. https://doi.org/10.1136/bmj.314.7080.570.

[20] Zhang Y. Fundamentals of Biostatistics[J]. The American Statistician, 2007, 61(2): 183-183. https://doi.org/10.1001/jama.1969.03160030112031.

[21] Bridwell K H, Baldus C, Berven S, et al. Changes in Radiographic and Clinical Outcomes With Primary Treatment Adult Spinal Deformity Surgeries From Two Years to Three-to Five-Years Follow-up[J]. Spine, 2010, 35(20): 1849-1854. https://doi.org/10.1097/BRS.0b013e3181 efa06a.

[22] Xia T, Long C R, Vining R D, et al. Association of lumbar spine stiffness and flexion-relaxation phenomenon with patient-reported outcomes in adults with chronic low back pain - a single-arm clinical trial investigating the effects of thrust spinal manipulation[J]. BMC Complement Altern Med, 2017, 17(1): 303. https://doi.org/10.1186/s12906-017-1821-1.

[23] Hamilton D K, Kong C, Hiratzka J, et al. Patient Satisfaction After Adult Spinal Deformity Surgery Does Not Strongly Correlate With Health-Related Quality of Life Scores, Radiographic Parameters, or Occurrence of Complications[J]. Spine (Phila Pa 1976), 2017, 42(10): 764-769. https://doi.org/10.1097/BRS.0000000000001921.

[24] Yuksel S, Ayhan S, Nabiyev V, et al. Minimum clinically important difference of the health-related quality of life scales in adult spinal deformity calculated by latent class analysis: is it appropriate to use the same values for surgical and nonsurgical patients?[J]. Spine J, 2018, 19(1): 71-78. https://doi.org/10.1016/j.spinee.2018.07.005.

[25] Wong A Y L, Kawchuk G N. The Clinical Value of Assessing Lumbar Posteroanterior Segmental Stiffness: A Narrative Review of Manual and Instrumented Methods[J]. PM R, 2016, 9(8): 816-830. https://doi.org/10.1016/j.pmrj.2016.12.001. 
[26] Taylor S J, Taylor A E, Foy M A, et al. Responsiveness of common outcome measures for patients with low back pain[J]. Spine (Phila Pa 1976), 1999, 24(17): 1805-1812.

https://doi.org/10.1097/00007632-199909010-00010.

[27] Wilk B, Karol L A, Johnston C E, 2nd, et al. The effect of scoliosis fusion on spinal motion: a comparison of fused and nonfused patients with idiopathic scoliosis[J]. Spine (Phila Pa 1976), 2006, 31(3): 309-314. https://doi.org/10.1097/01.brs.0000197168.11815.ec.

[28] Winter R B, Carr P, Mattson H L. A study of functional spinal motion in women after instrumentation and fusion for deformity or trauma[J]. Spine (Phila Pa 1976), 1997, 22(15): 1760-1764.

https://doi.org/10.1097/00007632-199708010-00016.

[29] Hart R A, Marshall L M, Hiratzka S L, et al. Functional limitations due to stiffness as a collateral impact of instrumented arthrodesis of the lumbar spine[J]. Spine (Phila Pa 1976), 2014, 39(24): E14681474. https://doi.org/10.1097/BRS.0000000000000595.

[30] Snodgrass S J, Haskins R, Rivett D A. A structured review of spinal stiffness as a kinesiological outcome of manipulation: Its measurement and utility in diagnosis, prognosis and treatment decisionmaking[J]. Journal of Electromyography and Kinesiology, 2012, 22(5): 708-723.

https://doi.org/10.1016/j.jelekin.2012.04.015.

[31] Glassman S D, Copay A G, Berven S H, et al. Defining substantial clinical benefit following lumbar spine arthrodesis[J]. J Bone Joint Surg Am, 2008, 90(9): 1839-1847.

https://doi.org/10.2106/JBJS.G.01095.

\section{Figures}


4

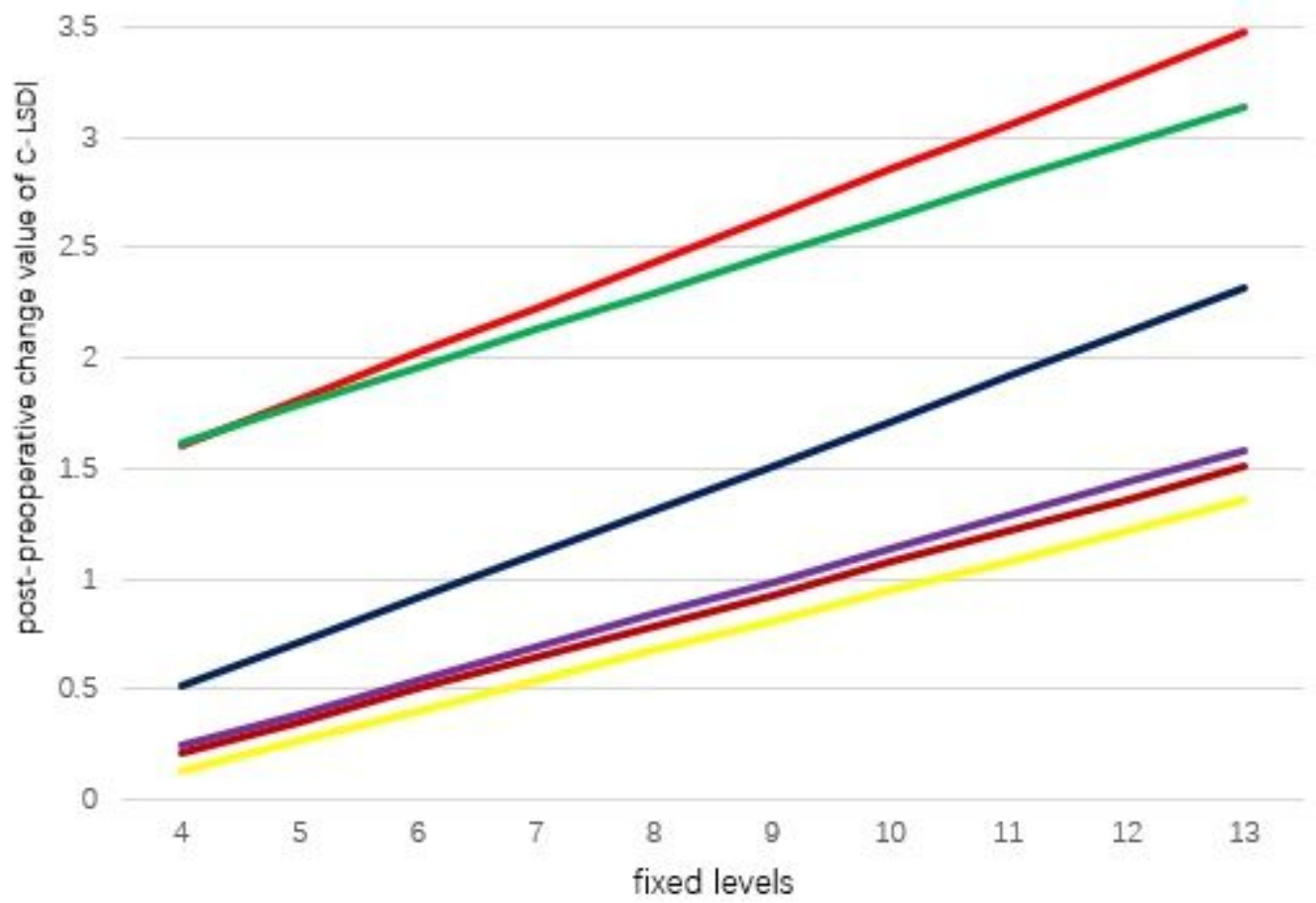

Bathe the lower half of your body on your own ( $P=0.006 * Y=0.200 \times X-0.286)$

Perform personal hygiene functions following toileting $(P=0.003 * Y=0.208 \times X+0.771)$

Bend forward to dean the floor on your own ( $P=0.021 * Y=0.149 \times X-0.354)$

Sit down on the chair and get up on your own ( $P=0.0497 * Y=0.136 \times X-0.415)$

Get in and out of a car on your own $(P=0.021 * Y=0.169 \times X+0.944)$

Lie down on the bed and get up on your own ( $P=0.022 * Y=0.144 \times X-0.366)$

\section{Figure 1}

Linear correlation between fixed levels and the change scores of C-LSDI. Fixed levels and several items in C-LSDI were positively correlated, and with increasing fixed levels, patients behaved significant stiffness in these activities. $Y=$ change scores of C-LSDI postoperatively $\mathbb{X}=$ fixed levels. 